\title{
Experimental Models and Correlations between Surface Parameters after Slide Diamond Burnishing
}

\author{
Mieczyslaw Korzynski ${ }^{1}$, Kazimiera Dudek ${ }^{1}$, Arkadiusz Palczak ${ }^{1}$, Bartosz Kruczek ${ }^{1}$, Paweł Kocurek ${ }^{2}$ \\ ${ }^{1}$ Centre for Innovative Technologies, University of Rzeszow, Pigonia 1, 35-310 Rzeszow, Poland, mk@ur.edu.pl \\ ${ }^{2}$ Mechanical Engineering and Aeronautics Faculty, Rzeszow University of Technology, W. Pola 2, 35-959 Rzeszow, Poland
}

\begin{abstract}
In the paper the set of representative parameters for a comprehensive assessment of the surface texture status after slide burnishing has been proposed. The analysis of correlations between the parameters of the surface texture, obtained by slide diamond burnishing of 317Ti steel has been performed. Correlations have been determined and several groups of surface texture parameters with strong mutual correlations (also parameters uncorrelated with the other) have been selected. For both groups of parameters - representative and uncorrelated - experimental mathematical relations defining influences of the input parameters of slide diamond burnishing on the surface texture parameters have been developed. Also, interaction effects for individual parameters of this finishing process have been disclosed. It has been found that by appropriate selection of input conditions of the slide diamond burnishing process, it is possible to obtain a wide range of states of the surface texture.
\end{abstract}

Keywords: Surface texture, finishing, slide diamond burnishing, 317Ti.

\section{INTRODUCTION}

Surface texture of the machine parts is the result of their finishing treatment. Often, the appropriate shaping of surface texture can radically change properties of the product, such as abrasion resistance, corrosion resistance, as well as their strength during changing operational loads. Therefore, various finishing techniques (inter alia slide diamond burnishing) are more and more developed and increasingly used. They are enabling to obtain optimal surface texture for the working conditions of given parts of machines. In such cases, the assessment of surface texture by conventional amplitude parameters is far from enough [1]-[3]. It's commonly found that the surfaces, having same values of the amplitude parameters, can have very different values of other surface texture parameters and thus different functional properties. Therefore, already in 1990, the surface texture has been evaluated on the basis of eight parameters [4]. According to later proposals, which are included on the so-called Birmingham List [5], [6], it is necessary to take measurements of 14 parameters of the surface texture, to estimate its properties. A slightly different opinion have the authors of paper [1], who believe, that a representative set of surface texture parameters should be different and should depend on the type of work of the particular surface.

According to the current ISO 25178-2 standard for the complete characterization of the surface texture, dozens of parameters should be measured. However, in industrial practice such a comprehensive characterization is never needed and a full assessment is never carried out. In the case of general-purpose, not very responsible machine parts, requirements are often set by determining the maximum permissible values of $R a / S a$ or other amplitude parameters [7]. In paper [8], measurements of the polymers wear were based on the $R a$ parameter. Meanwhile in [9], tests of the correlations between bearing vibrations and various surface parameters were made. It was stated that vibration level had had the highest correlation coefficient with $\mathrm{Sa} / \mathrm{Ra}$ parameters. However, in [10] it was found, that single $R a$ parameter is insufficient to describe the surface texture functionality. The optimization of hard turning process based on different stereometric parameters was made. Also in [7] there was surface state valuation based on different amplitude parameters, and in [11] roughness of the polished specimens was modelled with the use of $R a$ and $R y$ (maximum height of the profile) parameters.

In case of parts working in the friction conditions, numerous other parameters are also important. In work [12], there are 11 highlighted parameters that need to be considered during testing the correlation between surface texture and sliding friction. In [13], five amplitude parameters ( $R a, R q, R z, R k u$, and $R s k$ ) and five material ratio parameters ( $R k, R p k, R v k, M R 1$, and $M R 2$ ) were used to determine the best indicator of run-in. However, it follows from the study presented in [14] that one can fairly precisely describe a piston skirt surface topography using the following parameters: Sq, Ssk, Str, and Sdq. For the detailed description of topography, the authors of work [14] 
also recommend parameters $S t_{ \pm 3 \sigma}, S k u, S d s, S s c$, and $P \Delta a_{x} / P \Delta a_{y}$. Meanwhile in [15], tribological properties were evaluated in connection with six 3D parameters such as: $S a$, $S q$, St, Abbot-Firestone Curve, Texture Directivity, and 3D view of surface. In similar researches presented in [16], besides the Abbot-Firestone Curve and functional parameters, that came out of it, parameters $S q, S s c, S d s, S p d$ were also considered. Correlations between tribological properties and the state of surface, based on the 3D view of surface, and surface profile, but also $S z, S q, S s k, S k u, S t r$, Sal, Spd parameters, were described in [17]. In [18] the Ssk parameter was strongly correlated with the pressure, thickness of the lubrication layer in elastohydrodynamically lubricated sliding joints. The effect of surface geometry on the hydrodynamic bearing parameters was also shown in [19]. In [20] it was proven, that with usage of Ssk and Sku parameters it is possible to predict the contact region's tribological behavior. It was stated that higher Sku and negative Ssk value will end in lower friction and it was confirmed that both of Ssk and Sku parameters can be used to design the textured surface. Meanwhile in [21] the $R q, R z$, tp (bearing coefficient), Rsk parameters were used to describe the fabric's wear level. The spatial and hybrid stereometric parameters were used in [22] to estimate the surface topography in sliding friction conditions. In [23] after the tests of the surface roughness and texture parameters during lubricated sliding friction, there were few different specimens used. They were made with the isotropic and anisotropic surface roughness and with different textures. Thanks to these various specimens, the influence of the isotropic/anisotropic surface structure and Ssk and Sku parameters on the friction was proven. Also in [24] it was shown, that the tribological wear was correlated not only with $R a$ but also Ssk and Sku parameters.

The mentioned researches have varied approach to the problem. It also can be seen in the researches of the dynamic loaded surfaces. For example in [12], parameters $S a, S q, S t d$, and $S v i$ were considered as the most important. In [25], during fatigue tests, only $S q, S k$ parameters were verified and in [26] only $S a / R a$ and $S z / R z$ parameters and 3D view of surface were considered.

It is known and has been frequently found during experiments, that there are correlations between different surface texture parameters (including those not necessarily resulting from physical dependencies). For example, in [27], correlations between 21 surface texture parameters defined in the ISO 25178-2 standard have been found and during the examination of the 17 surfaces obtained as a result of different machining methods there was found the existence of many strong correlations between surface texture parameters. The existence of this kind of correlations has also been shown in [28], after examining the surfaces of the cylinder liners. And in [29] the correlations between different surface texture parameters have been described and a way of identifying parameters that are significantly changing during the manufacturing process has been proposed. The fact that some surface texture parameters are unnecessary because of redundancy has also been shown in [30].
Analysis of these dependencies enables determination of necessary requirements for the drawings of the state of machined surfaces, but also allows us to reduce the number of surface texture parameters that should be controlled during the production process. This kind of analysis for the slide diamond burnishing process of the cylindrical-shaped parts made of austenitic stainless steel has been carried out in this work. The work also includes the calculations of mathematical relations, defining the influence of the basic parameters of slide diamond burnishing on the surface texture properties.

\section{RESEARCH METHODOLOGY \& MEASURING TECHNIQUES}

The samples made of austenitic stainless steel 317Ti after drawing process have been used for the model studies. The samples were prepared (by cold drawing) in the shape of shafts, with a diameter of $12 \mathrm{~mm}$, a length of $100 \mathrm{~mm}$ and a surface roughness $S a=1.05 \mu \mathrm{m}$. Presented in Fig.1., slide burnishing process (this technology was described in detail in [31]) was performed on the universal lathe using a special chuck ensuring elastic and adjustable burnishing force. Onepiece spherical burnishers made of PCD (synthetic polycrystalline diamond also known as carbonado) have been used. During the burnishing process a lubricating mixture of oil and kerosene with the proportion of 20:80 has been used. Studies of influence of selected slide burnishing parameters on the surface roughness were carried out according to the Hartley plan PS/DS-P:Ha3 [32] (Table 1.). Calculation methodology was described in detail in [33].
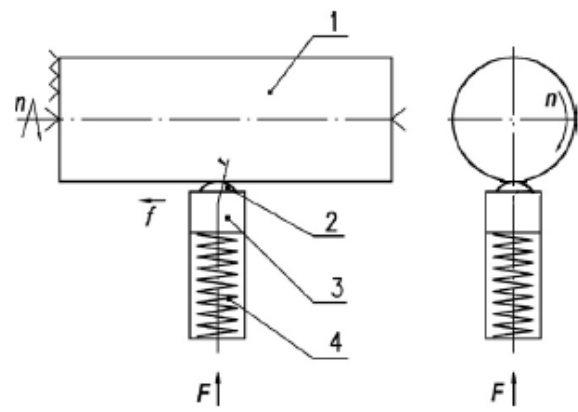

Fig.1. Slide diamond burnishing: 1 - object burnished,

2 - burnishing tool, 3 - tool holder, 4 - pressure control spring, $\mathrm{n}$ - rotational speed, $\mathrm{f}$ - feed, $\mathrm{F}$ - burnishing force.

Experiment required implementation of the $N=11$ tests, with input parameters on three levels of variation. Values of input parameters have been selected according to the previously acquired experience, technical capabilities of the test bench and the preliminary studies. The experiment was carried out with three repetitions. Regression analysis of the results was performed with the significance level of 0.05 . This allowed us to obtain mathematical relationships (regression equation), which describe the influence of the input parameters of the slide diamond burnishing process on the achieved results. The input parameters were: radius of the tool tip, load force, and tool feed. Output parameters were parameters of the surface texture obtained during the 
process. The values of input parameters used during the individual tests are given in Table 2.

Table 1. Matrix of the Hartley's plan PS/DS - P:Ha3.

\begin{tabular}{|c|c|c|c|c|c|c|c|c|c|}
\hline $\begin{array}{c}\text { Processing } \\
\text { variant }\end{array}$ & $\boldsymbol{x}_{1}$ & $\boldsymbol{x}_{2}$ & $\boldsymbol{x}_{3}$ & $\boldsymbol{x}_{1}{ }^{2}$ & $\boldsymbol{x}_{2}{ }^{2}$ & $\boldsymbol{x}_{3}{ }^{2}$ & $\boldsymbol{x}_{1} \boldsymbol{x}_{2}$ & $\boldsymbol{x}_{1} \boldsymbol{x}_{3}$ & $\boldsymbol{x}_{2} \boldsymbol{x}_{3}$ \\
\hline 1 & + & + & + & + & + & + & + & + & + \\
\hline 2 & + & - & - & + & + & + & - & - & + \\
\hline 3 & - & + & - & + & + & + & - & + & - \\
\hline 4 & - & - & + & + & + & + & + & - & - \\
\hline 5 & + & 0 & 0 & + & 0 & 0 & 0 & 0 & 0 \\
\hline 6 & - & 0 & 0 & + & 0 & 0 & 0 & 0 & 0 \\
\hline 7 & 0 & + & 0 & 0 & + & 0 & 0 & 0 & 0 \\
\hline 8 & 0 & - & 0 & 0 & + & 0 & 0 & 0 & 0 \\
\hline 9 & 0 & 0 & + & 0 & 0 & + & 0 & 0 & 0 \\
\hline 10 & 0 & 0 & - & 0 & 0 & + & 0 & 0 & 0 \\
\hline 11 & 0 & 0 & 0 & 0 & 0 & 0 & 0 & 0 & 0 \\
\hline
\end{tabular}

Table 2. Values of the input parameters for the experiments.

\begin{tabular}{|c|c|c|c|}
\hline $\begin{array}{c}\text { Input } \\
\text { parameters }\end{array}$ & $\begin{array}{c}\text { Tool tip radius } \\
\boldsymbol{r}[\mathbf{m m}]\end{array}$ & $\begin{array}{c}\text { Load force } \\
\boldsymbol{F}[\mathbf{N}]\end{array}$ & $\begin{array}{c}\text { Tool feed } \\
\boldsymbol{f}[\mathbf{m m} / \mathbf{r e v}]\end{array}$ \\
\hline Zero level (0) & 3 & 100 & 0.07 \\
\hline Upper level (+) & 4 & 150 & 0.11 \\
\hline Lower level (-) & 2 & 50 & 0.03 \\
\hline
\end{tabular}

Measurements of surface texture parameters were carried out on the Talyscan 150 profilometer with the software for surface analysis TalyMap Expert 2.0.15. The measurements were performed with the contact method, using inductive contact sensor equipped with the measuring needle with tip radius 5 microns. The set of 28 parameters given in ISO25178-2 and ISO-4287 standards was measured. With the use of results of these measurements (listed in Table 3.) Pearson's linear correlation coefficients between all studied parameters were calculated. The results of these calculations are given in the Table 4.

Table 3. Results of the surface texture measurements of tested samples.

\begin{tabular}{|c|c|c|c|c|c|c|c|c|c|c|c|c|}
\hline \multirow{2}{*}{\multicolumn{2}{|c|}{$\begin{array}{l}\text { Measured } \\
\text { parameter }\end{array}$}} & \multicolumn{11}{|c|}{ Processing variants } \\
\hline & & \multirow{2}{*}{\begin{tabular}{|c|}
$\mathbf{1}$ \\
0.153
\end{tabular}} & \multirow{2}{*}{$\begin{array}{c}\mathbf{2} \\
0.209\end{array}$} & \multirow{2}{*}{\begin{tabular}{|c|}
$\mathbf{3}$ \\
0.349
\end{tabular}} & \multirow{2}{*}{\begin{tabular}{|c|}
4 \\
0.341
\end{tabular}} & \multirow{2}{*}{\begin{tabular}{|c|}
$\mathbf{5}$ \\
0.206
\end{tabular}} & \multirow{2}{*}{\begin{tabular}{|c|}
$\mathbf{6}$ \\
0.234 \\
\end{tabular}} & \multirow{2}{*}{\begin{tabular}{|c|}
7 \\
0.145 \\
\end{tabular}} & \multirow{2}{*}{\begin{tabular}{|c|}
$\mathbf{8}$ \\
0.2
\end{tabular}} & \multirow{2}{*}{\begin{tabular}{|c|}
$\mathbf{9}$ \\
0.216
\end{tabular}} & \multirow{2}{*}{\begin{tabular}{|c|}
$\mathbf{1 0}$ \\
0.12
\end{tabular}} & \multirow{2}{*}{\begin{tabular}{|c|}
$\mathbf{1 1}$ \\
0.156
\end{tabular}} \\
\hline 1 & Sa & & & & & & & & & & & \\
\hline 2 & $S q$ & 0.262 & 0.435 & 0.496 & 0.616 & 0.438 & 0.313 & 0.211 & 0.416 & 0.291 & 0.189 & 0.256 \\
\hline 3 & $S p$ & 3.16 & 2.22 & 4.47 & 2.04 & 2.42 & 2.84 & 2.59 & 3.83 & 1.35 & 1.23 & 2.13 \\
\hline 4 & $S v$ & 6.19 & 8.61 & 5.82 & 16.2 & 8.54 & 4.53 & 4.79 & 11.1 & 6.95 & 7.44 & 8.52 \\
\hline 5 & $S t$ & 9.39 & 10.8 & 10.3 & 18.3 & 11 & 7.37 & 7.38 & 14.9 & 8.31 & 8.67 & 10.7 \\
\hline 6 & Ssk & -6.04 & -6.8 & -0.954 & -7.31 & -7.79 & -0.748 & -2.99 & -9.91 & -2.43 & -7.17 & -8.82 \\
\hline 7 & Sku & 83.3 & 77 & 9.27 & 115 & 99.5 & 6.84 & 41.5 & 179 & 36 & 158 & 197 \\
\hline 8 & $S z$ & 7.22 & 8.92 & 8.75 & 12.1 & 9.63 & 5.16 & 5.02 & 11.3 & 5.02 & 4.58 & 7.54 \\
\hline 9 & $S T p$ & 57.2 & 61.1 & 56.6 & 56.4 & 57.8 & 53.7 & 52.3 & 55.1 & 50.5 & 53.2 & 52.8 \\
\hline 10 & Smmr & 0.00619 & 0.00861 & 0.00582 & \begin{tabular}{|l|}
0.0162 \\
\end{tabular} & 0.00854 & 0.00453 & 0.00479 & \begin{tabular}{|l|}
0.0111 \\
\end{tabular} & 0.00695 & 0.00744 & 0.00852 \\
\hline 11 & Smvr & 0.00316 & 0.00222 & 0.00447 & 0.00204 & 0.00242 & 0.00284 & 0.00259 & 0.00383 & 0.00135 & 0.00123 & 0.00213 \\
\hline 12 & $S P c$ & 213 & 190 & 315 & \begin{tabular}{|l|}
88.5 \\
\end{tabular} & 208 & 299 & 220 & 235 & \begin{tabular}{|l|}
77.1 \\
\end{tabular} & 288 & 144 \\
\hline 13 & Sds & 1823 & 1852 & 1486 & 1595 & 1887 & 1835 & 1791 & 2315 & 1168 & 2225 & 1482 \\
\hline 14 & Str & 0.447 & 0.549 & 0.0872 & 0.563 & 0.858 & 0.264 & 0.218 & 0.634 & 0.0558 & 0.545 & 0.213 \\
\hline 15 & Sal & 0.0437 & 0.0421 & 0.0285 & 0.0706 & 0.0892 & 0.0475 & 0.0316 & 0.076 & 0.0312 & 0.115 & 0.035 \\
\hline 16 & Std & 22 & 45 & 2 & 63.5 & 26.5 & 1.5 & 88 & 45 & 88.5 & 45 & 63.5 \\
\hline 17 & $S f d$ & 2.13 & 2.06 & 2.38 & 2.1 & 2.03 & 2.38 & 2.27 & 2.08 & 2.23 & 2.12 & 2.14 \\
\hline 18 & $S d q$ & 0.0355 & 0.0484 & 0.104 & 0.0495 & 0.0399 & 0.0624 & 0.0306 & 0.037 & 0.0291 & 0.0213 & 0.0284 \\
\hline 19 & SsC & \begin{tabular}{|l|}
0.00562 \\
\end{tabular} & 0.00557 & 0.0148 & \begin{tabular}{|l}
0.00698 \\
\end{tabular} & 0.00542 & 0.00976 & 0.00599 & 0.00515 & 0.00582 & 0.00444 & 0.00545 \\
\hline 20 & $S d r$ & 0.0623 & \begin{tabular}{|l|}
0.115 \\
\end{tabular} & 0.536 & \begin{tabular}{|l|}
0.121 \\
\end{tabular} & 0.078 & \begin{tabular}{|l|}
0.193 \\
\end{tabular} & \begin{tabular}{|l|}
0.0466 \\
\end{tabular} & \begin{tabular}{|l|}
0.0669 \\
\end{tabular} & \begin{tabular}{|l|}
0.0421 \\
\end{tabular} & \begin{tabular}{|l|}
0.0226 \\
\end{tabular} & \begin{tabular}{|l|}
0.04 \\
\end{tabular} \\
\hline 21 & Sbi & 0.0913 & 0.238 & 0.131 & 0.442 & 0.221 & 0.131 & 0.0919 & 0.122 & 0.315 & 0.193 & 0.141 \\
\hline 22 & Sci & 0.96 & 0.739 & 1.22 & 0.929 & 0.864 & 1.32 & 1.28 & 0.917 & 1.39 & 1.2 & 1.12 \\
\hline 23 & Svi & 0.149 & 0.165 & 0.167 & 0.141 & 0.146 & 0.152 & 0.137 & 0.122 & 0.113 & 0.121 & 0.119 \\
\hline 24 & $S k$ & 0.421 & 0.455 & 0.865 & 0.914 & 0.46 & 0.7 & 0.443 & 0.512 & 0.706 & 0.347 & 0.479 \\
\hline 25 & Spk & 0.164 & 0.237 & 0.496 & 0.447 & 0.305 & 0.273 & 0.179 & 0.398 & 0.208 & 0.159 & 0.173 \\
\hline 26 & Svk & 0.465 & 0.903 & 0.834 & 0.995 & 0.808 & 0.458 & 0.322 & 0.672 & 0.381 & 0.239 & 0.337 \\
\hline 27 & Sr1 & 8.79 & 11.7 & 11.4 & 9.17 & 13.2 & 9.03 & 9.28 & 10.3 & 9.55 & 10.2 & 8.99 \\
\hline 28 & Sr2 & 87.9 & 86.6 & 83.8 & 87.3 & 87.2 & 87.6 & 89.9 & 89.4 & 93 & 88.4 & 90.2 \\
\hline
\end{tabular}




\begin{tabular}{|c|c|c|c|c|c|c|c|c|c|c|c|c|c|c|c|c|c|c|c|c|c|c|c|c|c|c|c|c|}
\hline ம் & $\neg$ & & & & & & & & & & & & & & & & & & & & & & & & & & & \\
\hline$\overline{5}$ & $\begin{array}{c}1 \\
0 \\
0 \\
0\end{array}$ & - & & & & & & & & & & & & & & & & & & & & & & & & & & \\
\hline के & $\begin{array}{l}\stackrel{9}{9} \\
0\end{array}$ & 节 & $r$ & & & & & & & & & & & & & & & & & & & & & & & & & \\
\hline के & \begin{tabular}{c}
\multirow{2}{*}{} \\
o
\end{tabular} & $\begin{array}{ll}\infty \\
0 \\
0 \\
0\end{array}$ & \begin{tabular}{|l|}
0 \\
0 \\
0 \\
1
\end{tabular} & - & & & & & & & & & & & & & & & & & & & & & & & & \\
\hline$\dot{\omega}$ & 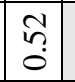 & \begin{tabular}{|l|} 
\\
\\
0 \\
0
\end{tabular} & $\frac{m}{3}$ & \&̊. & $\neg$ & & & & & & & & & & & & & & & & & & & & & & & \\
\hline$\frac{\vec{\omega}}{\omega}$ & $\begin{array}{l}\tilde{O} \\
\tilde{0}\end{array}$ & 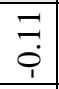 & $\begin{array}{l}\infty \\
\stackrel{1}{0} \\
0\end{array}$ & $\begin{array}{l}+ \\
0 \\
0 \\
\end{array}$ & 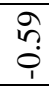 & -1 & & & & & & & & & & & & & & & & & & & & & & \\
\hline के & 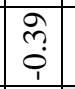 & \begin{tabular}{|l|}
$\infty$ \\
0 \\
0 \\
\end{tabular} & $\begin{array}{l}\text { Nָ } \\
\text { Q } \\
\end{array}$ & 荬 & fे & $\begin{array}{l}\text { ô } \\
\text { के }\end{array}$ & - & & & & & & & & & & & & & & & & & & & & & \\
\hline के & 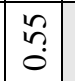 & $\begin{array}{l}0 \\
\infty \\
0\end{array}$ & $\begin{array}{l}0 \\
\text { mి } \\
0\end{array}$ & $\begin{array}{l}2 \\
\hat{0} \\
0\end{array}$ & б. & $\begin{array}{l}\text { पn } \\
\text { in }\end{array}$ & ֻू & -1 & & & & & & & & & & & & & & & & & & & & \\
\hline Es & స̃ & $\begin{array}{l}\infty \\
\substack{c \\
0 \\
0}\end{array}$ & ๙ิ & సิ & 仓ิ & 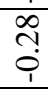 & $\begin{array}{l}\sigma \\
\dot{0} \\
\end{array}$ & $\begin{array}{l}0 . \\
0\end{array}$ & - & & & & & & & & & & & & & & & & & & & \\
\hline है & $\begin{array}{l}\stackrel{g}{q} \\
0\end{array}$ & $\begin{array}{l}\infty \\
0 \\
0 \\
0\end{array}$ & $\begin{array}{l}0 \\
-1 \\
0 \\
1\end{array}$ & - & $\begin{array}{l}\mathscr{H} \\
0\end{array}$ & $\begin{array}{l}\text { to } \\
\stackrel{0}{1}\end{array}$ & 苞 & $\begin{array}{l}\stackrel{0}{0} \\
\hat{0}\end{array}$ & సิ & $r$ & & & & & & & & & & & & & & & & & & \\
\hline है & $\begin{array}{l}9 \\
\stackrel{9}{0}\end{array}$ & 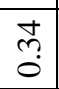 & - & $\begin{array}{l}0 \\
0 \\
0 \\
1\end{array}$ & $\stackrel{m}{0}$ & $\begin{array}{l}\infty \\
\stackrel{1}{0} \\
\end{array}$ & $\begin{array}{l}\text { ज̂ } \\
\text { î }\end{array}$ & ले & ஸึ. & $\begin{array}{l}0 \\
0 \\
1 \\
1\end{array}$ & -1 & & & & & & & & & & & & & & & & & \\
\hline$\frac{v}{\omega}$ & $\begin{array}{l}\hat{0} \\
\dot{i} \\
\text { in }\end{array}$ & $\begin{array}{l}9 \\
\stackrel{2}{0} \\
\dot{9}\end{array}$ & 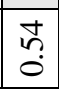 & 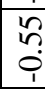 & $\begin{array}{l}\text { के } \\
\text { m. } \\
\text { in }\end{array}$ & $\begin{array}{l}\text { त्र } \\
\text { o }\end{array}$ & $\begin{array}{l}\text { הี } \\
\text { ָे }\end{array}$ & $\begin{array}{l}\text { Nै } \\
\text { in } \\
\end{array}$ & $\stackrel{\nabla}{\stackrel{D}{0}}$ & 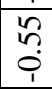 & 苞 & -1 & & & & & & & & & & & & & & & & \\
\hline$\ddot{n}$ & $\begin{array}{l}\vec{F} \\
\overrightarrow{0} \\
\dot{1}\end{array}$ & \begin{tabular}{l}
7 \\
\multirow{1}{*}{} \\
\end{tabular} & $\begin{array}{ll}10 \\
0 \\
0\end{array}$ & ô. & 궁 & 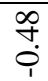 & $\stackrel{m}{0}$ & $\stackrel{\vec{J}}{-}$ & సิ & $\hat{\circ}$ & 늠 & 婴 & - & & & & & & & & & & & & & & & \\
\hline$\dot{\omega}$ & $\begin{array}{l}0 \\
\stackrel{m}{0} \\
\\
1\end{array}$ & 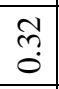 & \begin{tabular}{|l|}
$\infty$ \\
0 \\
0 \\
0
\end{tabular} & $\begin{array}{l}\text { 우 } \\
0 \\
\end{array}$ & $\begin{array}{l}\infty \\
\stackrel{\infty}{+} \\
0\end{array}$ & $\begin{array}{l}\overrightarrow{\hat{N}} \\
0 \\
i\end{array}$ & $\begin{array}{l}\stackrel{\infty}{+} \\
\stackrel{0}{0} \\
\end{array}$ & 苞 & ڤึ? & 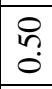 & $\begin{array}{l}\infty \\
0 \\
0 \\
1\end{array}$ & 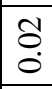 & $\begin{array}{l}0 \\
0 \\
0 \\
0\end{array}$ & - & & & & & & & & & & & & & & \\
\hline$\overline{\tilde{E}}$ & 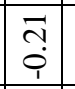 & $\begin{array}{l} \\
\\
0\end{array}$ & $\begin{array}{l}\stackrel{0}{0} \\
\text { m. } \\
\text { i }\end{array}$ & $\begin{array}{c}\text { के } \\
\text { o. } \\
0\end{array}$ & $\begin{array}{l}\text { స్ } \\
\text { Oे }\end{array}$ & $\begin{array}{l}\text { 员 } \\
0 \\
\\
\end{array}$ & 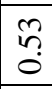 & $\stackrel{\infty}{\rightarrow}$ & $\begin{array}{l}\circ \\
\stackrel{\circ}{\circ} \\
0\end{array}$ & $\begin{array}{c}\text { m. } \\
\text { m. } \\
\text {. }\end{array}$ & $\begin{array}{l}\text { mे } \\
\text { i. } \\
\end{array}$ & $\stackrel{9}{0}$ & $\begin{array}{l}0 \\
0 \\
0\end{array}$ & $\begin{array}{l}\hat{\imath} \\
0\end{array}$ & - & & & & & & & & & & & & & \\
\hline$\Xi$ & $\begin{array}{l}0 \\
\text { mे } \\
0 \\
i\end{array}$ & 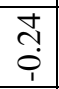 & 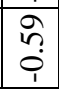 & 뇸 & $\begin{array}{l}\mathscr{D}^{\circ} \\
\stackrel{0}{0}\end{array}$ & $\begin{array}{l}\overrightarrow{\tilde{N}} \\
\text { î }\end{array}$ & $\begin{array}{l}\text { Nָ } \\
\text { ஸे }\end{array}$ & $\begin{array}{l}\text { J } \\
\stackrel{1}{0} \\
\end{array}$ & $\begin{array}{l}\text { 我 } \\
\stackrel{+}{+}\end{array}$ & 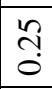 & 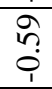 & $\begin{array}{l}\widetilde{N} \\
\hat{N} \\
\end{array}$ & \begin{tabular}{l}
$\infty$ \\
\multirow{1}{*}{} \\
1 \\
1
\end{tabular} & - & 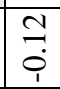 & - & & & & & & & & & & & & \\
\hline $\bar{s}$ & $\vec{m}$ & \begin{tabular}{|c|} 
\\
0 \\
0 \\
1 \\
1
\end{tabular} & 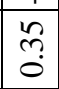 & $\begin{array}{l}\vec{b} \\
0 \\
\end{array}$ & 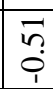 & б. & \begin{tabular}{l}
$\mathbb{N}$ \\
\multirow{1}{0}{}
\end{tabular} & $\begin{array}{l}\text { क्ष } \\
\stackrel{9}{0} \\
\end{array}$ & $\begin{array}{l}\text { Fै } \\
\stackrel{1}{0}\end{array}$ & $\begin{array}{l}\overrightarrow{0} \\
\dot{0}\end{array}$ & 留 & ปั & $\begin{array}{l}1 \\
0 \\
0 \\
0\end{array}$ & $\begin{array}{l}1 \\
0 \\
0 \\
0 \\
\end{array}$ & 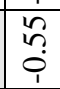 & \begin{tabular}{|l} 
\\
$\stackrel{2}{2}$ \\
0 \\
\end{tabular} & $\neg$ & & & & & & & & & & & \\
\hline$\overline{5}$ & \begin{tabular}{l} 
\\
\hdashline \\
0 \\
0
\end{tabular} & 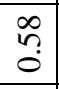 & $\begin{array}{l}\text { g) } \\
0 \\
0\end{array}$ & \begin{tabular}{l}
7 \\
\multirow{0}{*}{} \\
\end{tabular} & $\begin{array}{l}\infty \\
\stackrel{0}{0} \\
0\end{array}$ & 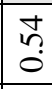 & 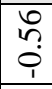 & స̂. & $\begin{array}{l}\text { Dे. } \\
\text { m. } \\
0\end{array}$ & $\begin{array}{l}\text { I. } \\
\text { - } \\
\text { i }\end{array}$ & $\begin{array}{l}0 \\
0 \\
0 \\
0\end{array}$ & \begin{tabular}{|l} 
\\
\\
\end{tabular} & 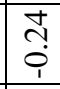 & 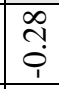 & $\begin{array}{l}0 \\
0 \\
0 \\
\\
\end{array}$ & \begin{tabular}{|l|}
0 \\
0 \\
0 \\
0 \\
\end{tabular} & 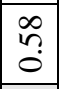 & - & & & & & & & & & & \\
\hline ڤ్ & $\begin{array}{l}\tilde{N} \\
\stackrel{0}{0}\end{array}$ & m & ఫ़ & $\begin{array}{l}\hat{N} \\
\text { ì }\end{array}$ & $\begin{array}{l}\text { ô. } \\
\text { i }\end{array}$ & $\begin{array}{l}\text { : } \\
0 \\
0\end{array}$ & $\begin{array}{l}\text { तै } \\
\stackrel{0}{0}\end{array}$ & $\begin{array}{l}8 \\
0 \\
0\end{array}$ & $\begin{array}{l}8 \\
0\end{array}$ & $\begin{array}{l}\text { त̂̀ } \\
\text { ì }\end{array}$ & $\begin{array}{l}\text { पे } \\
0\end{array}$ & $\begin{array}{l}0 \\
0 \\
0\end{array}$ & $\begin{array}{l}\text { ñ } \\
\text { m. } \\
0\end{array}$ & $\begin{array}{l}\text { o } \\
\text { †े } \\
\end{array}$ & $\begin{array}{l}\text { Iै } \\
\text { ô }\end{array}$ & \begin{tabular}{|l|}
$\infty$ \\
1 \\
0 \\
0 \\
\end{tabular} & $\begin{array}{l}\hat{0} \\
\hat{0}\end{array}$ & $\begin{array}{l}\text { 어 } \\
\end{array}$ & -1 & & & & & & & & & \\
\hline ఉ & $\begin{array}{l}\text { t } \\
\stackrel{0}{0}\end{array}$ & $\stackrel{\substack{0 \\
0 \\
0}}{0}$ & $\stackrel{\infty}{0}$ & $\begin{array}{l}\text { 我 } \\
\stackrel{1}{1}\end{array}$ & न. & $\begin{array}{l}\text { L̊ } \\
\text { L }\end{array}$ & 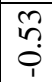 & न & ָิ & $\begin{array}{l}\frac{9}{7} \\
\stackrel{1}{1}\end{array}$ & $\begin{array}{l}\infty \\
0 \\
0 \\
0\end{array}$ & 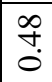 & 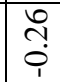 & \begin{tabular}{|c} 
\\
0 \\
0 \\
\end{tabular} & $\begin{array}{c}\text { m. } \\
\text { ọ } \\
\end{array}$ & \begin{tabular}{l|}
$\overrightarrow{0}$ \\
0 \\
\end{tabular} & $\begin{array}{l}\tilde{S} \\
0 \\
0\end{array}$ & \begin{tabular}{|l|} 
\\
0 \\
0
\end{tabular} & बे & -1 & & & & & & & & \\
\hline के & $\begin{array}{l}\stackrel{\infty}{+} \\
0\end{array}$ & $\begin{array}{l}\hat{n} \\
0 \\
0\end{array}$ & $\begin{array}{l}\text { 䓒 } \\
\text { Q }\end{array}$ & $\widehat{N}$ & นُ & $\begin{array}{l} \pm \\
\stackrel{9}{1}\end{array}$ & ¿ & 仓ิ & : & $\stackrel{\mathbb{N}}{0}$ & $\begin{array}{l}\text { 苟 } \\
\text { î }\end{array}$ & $\begin{array}{l}9 \\
0 \\
0 \\
1\end{array}$ & $\begin{array}{l}0 \\
0 \\
0 \\
0\end{array}$ & $\stackrel{9}{0}$ & $\overrightarrow{\tilde{o}}$ & \begin{tabular}{|l|} 
\\
0 \\
0 \\
0
\end{tabular} & $\begin{array}{c}\tilde{2} \\
\tilde{n} \\
\hat{\varphi} \\
\end{array}$ & \begin{tabular}{|l|} 
\\
0 \\
0 \\
\end{tabular} & $\begin{array}{l} \pm \\
\stackrel{0}{0}\end{array}$ & \begin{tabular}{c}
7 \\
\multirow{1}{*}{} \\
$i$
\end{tabular} & -1 & & & & & & & \\
\hline $\bar{\omega}$ & $\begin{array}{l}0 \\
0 \\
\dot{1} \\
\end{array}$ & \begin{tabular}{|l|}
$\vec{n}$ \\
0 \\
\end{tabular} & \begin{tabular}{|l|} 
\\
0 \\
0 \\
\end{tabular} & 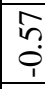 & $\begin{array}{l}\stackrel{8}{0} \\
\stackrel{0}{0}\end{array}$ & त् & $\begin{array}{l}\text { Iै } \\
\stackrel{+}{0}\end{array}$ & $\begin{array}{l}\frac{1}{\Omega} \\
\stackrel{9}{9}\end{array}$ & $\begin{array}{l}0 \\
\infty \\
0 \\
\end{array}$ & $\begin{array}{l}\text { 命 } \\
\text { î }\end{array}$ & $\begin{array}{l}m \\
? \\
\stackrel{0}{1}\end{array}$ & $\stackrel{10}{0}$ & $\begin{array}{l}\text { 우 } \\
\text { i }\end{array}$ & 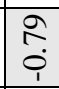 & $\begin{array}{l}\vec{m} \\
\dot{\rho} \\
\end{array}$ & \begin{tabular}{|c|}
0 \\
0 \\
0 \\
\end{tabular} & $\begin{array}{l}\Omega \\
⿱ 乛 \\
0\end{array}$ & \begin{tabular}{|l|} 
\\
0 \\
0 \\
\end{tabular} & \begin{tabular}{c}
$m$ \\
\hdashline \\
0
\end{tabular} & $\begin{array}{l}0 \\
\\
0\end{array}$ & \begin{tabular}{|c|} 
\\
$\stackrel{9}{1}$ \\
\\
1
\end{tabular} & $\neg$ & & & & & & \\
\hline डे & 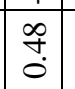 & $\begin{array}{l}0 \\
\stackrel{0}{0} \\
0\end{array}$ & กิ & 궁 & $\begin{array}{l}\text { जै } \\
\text { Oे }\end{array}$ & $\begin{array}{l}\text { mo } \\
\text { m. }\end{array}$ & $\begin{array}{l}\text { 全 } \\
\text { ? }\end{array}$ & ָั & $\begin{array}{l}\stackrel{0}{2} \\
\stackrel{0}{0}\end{array}$ & $\begin{array}{l}\text { जे } \\
\text { : }\end{array}$ & గ్ర & f & $\begin{array}{l}8 \\
0 \\
0\end{array}$ & \begin{tabular}{|l|} 
\\
0 \\
0
\end{tabular} & \begin{tabular}{l}
0 \\
\multirow{1}{*}{} \\
$\underline{1}$
\end{tabular} & \begin{tabular}{|l|}
0 \\
0 \\
0 \\
0
\end{tabular} & 芩 & \begin{tabular}{|l|}
\multirow{2}{*}{} \\
\end{tabular} & 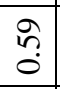 & $\begin{array}{l}0 \\
\bullet \\
0 \\
0\end{array}$ & \begin{tabular}{l}
7 \\
\multirow{1}{*}{} \\
$\dot{0}$ \\
1
\end{tabular} & $\begin{array}{l} \\
\text { m. } \\
\vdots \\
\end{array}$ & - & & & & & \\
\hline फे & 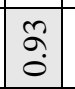 & $\begin{array}{l}\infty \\
0 \\
0 \\
0\end{array}$ & $\stackrel{\text { Ln }}{\stackrel{0}{0}}$ & 尚 & ले & $\begin{array}{l}\text { fo } \\
\text { !. }\end{array}$ & \begin{tabular}{l}
\multirow{J}{*}{} \\
$\stackrel{i}{0}$
\end{tabular} & $\stackrel{m}{0}$ & $\begin{array}{l}\text { पे } \\
\stackrel{0}{0}\end{array}$ & $\begin{array}{c}\tilde{n} \\
\tilde{0}\end{array}$ & ํㅠㅁ & $\begin{array}{l}9 \\
\\
0 \\
\end{array}$ & $\begin{array}{l}0 \\
\text { Lึ } \\
0 \\
\end{array}$ & $\begin{array}{l}\hat{n} \\
\hat{\rho} \\
\end{array}$ & $\begin{array}{l}\vec{m} \\
\dot{p} \\
\end{array}$ & $\begin{array}{l}0 \\
0 \\
0 \\
\end{array}$ & \begin{tabular}{l}
\multirow{2}{*}{} \\
\\
\end{tabular} & \begin{tabular}{|l|}
$\infty$ \\
0 \\
0 \\
0
\end{tabular} & $\begin{array}{l}8 \\
0 \\
0\end{array}$ & $\begin{array}{l}\tilde{B} \\
\dot{0} \\
0\end{array}$ & 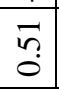 & 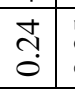 & \begin{tabular}{l}
\multirow{2}{*}{} \\
\hdashline \\
0
\end{tabular} & - & & & & \\
\hline के & 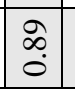 & $\begin{array}{c}\hat{1} \\
0 \\
0 \\
0\end{array}$ & $\begin{array}{l}\text { ị } \\
0 \\
0\end{array}$ & 今े & $\begin{array}{l}\text { : } \\
0 \\
0\end{array}$ & $\stackrel{\circ}{\circ}$ & $\begin{array}{l} \pm \\
\stackrel{1}{1}\end{array}$ & $\stackrel{m}{0}$ & ma & f̊ & $\stackrel{8}{8}$ & $\stackrel{1}{\circ}$ & $\begin{array}{l}\vec{d} \\
0 \\
\dot{0}\end{array}$ & F. & $\begin{array}{l}0 \\
0 \\
0\end{array}$ & \begin{tabular}{|l|}
0 \\
ñ \\
0 \\
\end{tabular} & 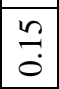 & \begin{tabular}{|l|}
\multirow{0}{*}{} \\
\end{tabular} & $\begin{array}{l}\tilde{\theta} \\
0 \\
0\end{array}$ & $\begin{array}{l} \\
\dot{8} \\
0\end{array}$ & ָָ & $\begin{array}{l}\vec{N} \\
\stackrel{1}{0} \\
\end{array}$ & 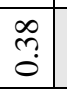 & $\begin{array}{l}\stackrel{n}{2} \\
\stackrel{0}{0}\end{array}$ & $\rightarrow$ & & & \\
\hline$\frac{\pi}{\vec{a}}$ & $\begin{array}{l}0 \\
\stackrel{0}{0} \\
0\end{array}$ & $\begin{array}{l}0 \\
0 \\
0\end{array}$ & $\begin{array}{l}\mathscr{0} \\
0 \\
0\end{array}$ & 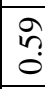 & $\begin{array}{l}\text { : } \\
0 \\
0\end{array}$ & $\begin{array}{l}\text { L } \\
-1 \\
0 \\
\end{array}$ & $\begin{array}{l}\overrightarrow{7} \\
\\
\end{array}$ & 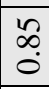 & $\hat{\imath}$ & \begin{tabular}{|l|}
\multirow{2}{0}{} \\
ஸุ \\
\end{tabular} & $\begin{array}{l}0 \\
\text { లె } \\
0\end{array}$ & \begin{tabular}{ll} 
\\
\multirow{1}{*}{} \\
\end{tabular} & $\begin{array}{l}0 \\
0 \\
0 \\
\end{array}$ & รั) & $\begin{array}{l}0 \\
0 \\
0\end{array}$ & \begin{tabular}{|l|}
\multirow{2}{*}{} \\
\multirow{1}{*}{} \\
\end{tabular} & 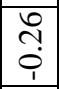 & 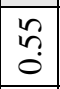 & $\begin{array}{c}\tilde{N} \\
0 \\
0\end{array}$ & $\stackrel{\text { L }}{8}$ & 今े & $\begin{array}{l}\hat{0} \\
\dot{0} \\
i\end{array}$ & $\begin{array}{l}\overrightarrow{0} \\
\dot{0}\end{array}$ & \begin{tabular}{l}
\multirow{2}{*}{} \\
ధุ \\
\end{tabular} & $\hat{\widehat{0}}$ & - & & \\
\hline$\overline{5}$ & 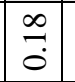 & $\begin{array}{l}9 \\
\stackrel{9}{0} \\
\end{array}$ & $\begin{array}{ll}0 \\
0 \\
0\end{array}$ & $\begin{array}{l}\tilde{O} \\
0 \\
0\end{array}$ & $\hat{\circ}$ & $\begin{array}{ll} \\
\\
0 \\
\end{array}$ & \begin{tabular}{|l|}
\multicolumn{2}{l}{} \\
0 \\
0 \\
\end{tabular} & ले & 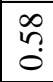 & $\begin{array}{l}\tilde{m} \\
\dot{0}\end{array}$ & $\begin{array}{l}\text { Ln } \\
\text { ?- }\end{array}$ & 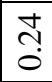 & $\begin{array}{l}\text { กิ } \\
\text { ○े }\end{array}$ & $\begin{array}{l}\text { 号 } \\
\text { O }\end{array}$ & $\vec{m}$ & \begin{tabular}{|l|}
$\vec{m}$ \\
$\dot{\rho}$ \\
$i$
\end{tabular} & 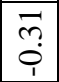 & \begin{tabular}{|c|}
\multirow{2}{*}{} \\
\multirow{0}{0}{} \\
\end{tabular} & $\overrightarrow{7}$ & 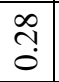 & $\begin{array}{l} \\
0 \\
0\end{array}$ & \begin{tabular}{l|l}
0 \\
0 \\
0 \\
1
\end{tabular} & \begin{tabular}{l|l|}
\multirow{m}{*}{} \\
\multirow{0}{*}{}
\end{tabular} & $\begin{array}{l}\overrightarrow{7} \\
\overrightarrow{0} \\
i\end{array}$ & $\begin{array}{c}\tilde{m} \\
\tilde{0}\end{array}$ & $\begin{array}{l}\text { 苞 } \\
0\end{array}$ & - & \\
\hline พิ & 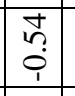 & 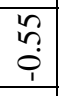 & \begin{tabular}{|l} 
\\
Ln \\
0 \\
\end{tabular} & $\begin{array}{l}0 \\
0 \\
0 \\
\end{array}$ & $\begin{array}{l}\text { त्र } \\
\text { î }\end{array}$ & $\begin{array}{l}m \\
0 \\
\end{array}$ & $\begin{array}{l}\text { N } \\
0 \\
\end{array}$ & $\begin{array}{l}\text { P̊+ } \\
\text { i }\end{array}$ & $\begin{array}{l}\text { Na } \\
\hat{0} \\
\end{array}$ & $\begin{array}{l}\text { mo } \\
\dot{0} \\
\end{array}$ & 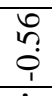 & $\begin{array}{l}\text { जि } \\
\text { i़ }\end{array}$ & $\begin{array}{l}\text { N̦ } \\
\text { in } \\
\end{array}$ & 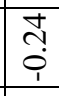 & $\begin{array}{l}8 \\
0 \\
0 \\
\end{array}$ & $\begin{array}{l}\hat{A} \\
0 \\
0\end{array}$ & \begin{tabular}{|c|} 
\\
\\
\\
\\
\end{tabular} & 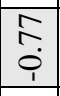 & $\begin{array}{l}\tilde{6} \\
0 \\
i \\
\end{array}$ & \begin{tabular}{l} 
\\
\multirow{2}{*}{} \\
$i$ \\
$i$
\end{tabular} & \begin{tabular}{|l|} 
\\
\\
0 \\
\end{tabular} & 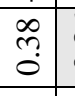 & \begin{tabular}{l|}
0 \\
0 \\
0 \\
1 \\
\end{tabular} & 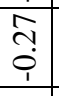 & $\begin{array}{l}\hat{u} \\
\hat{\rho} \\
\end{array}$ & $\begin{array}{l}0 \\
0 \\
0 \\
i \\
\end{array}$ & \begin{tabular}{|l|}
\multirow{0}{*}{} \\
\end{tabular} & -1 \\
\hline & $\mathscr{\omega}$ & ๘ & के & के & $\tilde{\omega}$ & らँ & 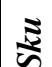 & సै & $\vec{E}$ & $\Xi$ & 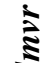 & รี & కే & $\$$ & $\overline{\tilde{s}}$ & $\Xi$ & $\frac{\delta}{5}$ & $\frac{5}{5}$ & $\begin{array}{l}\tilde{D} \\
\tilde{\omega}\end{array}$ & 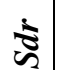 & $\ddot{\tilde{\omega}}$ & $\tilde{\dot{\omega}}$ & $\tilde{\omega}$ & ఉ & $\frac{\pi}{2}$ & के & $\bar{s}$ & $\tilde{\kappa}$ \\
\hline
\end{tabular}




\section{DisCUSSION OF TEST RESUlTS}

If we consider correlation coefficients with absolute value greater than 0.7 as significant (highlighted in the Table 4.), it can be concluded, that there are many dependences between the surface texture parameters after the slide burnishing process. Most parameters are correlated with one of the following: Sa, Sv, Str, Ssk, Svk, Std, Svi or Sp. These parameters were found to be representative for the most of the others, as it was shown in Table 5 . It also revealed parameters, which have been very poorly correlated with the others: Sds and $\operatorname{Sr} 1$.

Table 5. Correlation coefficient values of surface texture parameters.

\begin{tabular}{|c|c|c|}
\hline $\begin{array}{c}\text { Representative } \\
\text { parameter }\end{array}$ & $\begin{array}{l}\text { Correlated } \\
\text { parameters }\end{array}$ & $\begin{array}{l}\text { Correlation } \\
\text { coefficient value }\end{array}$ \\
\hline \multirow{4}{*}{ Sa } & $S d q$ & 0.80 \\
\cline { 2 - 3 } & $S s c$ & 0.73 \\
\cline { 2 - 3 } & $S d r$ & 0.74 \\
\cline { 2 - 3 } & $S k$ & 0.93 \\
\cline { 2 - 3 } & $S p k$ & 0.89 \\
\cline { 2 - 3 } & $S t$ & 0.96 \\
\cline { 2 - 3 } Sv & $S z$ & 0.91 \\
\cline { 2 - 3 } & $S m m r$ & 1 \\
\cline { 2 - 3 } & $S b i$ & 0.72 \\
\hline \multirow{4}{*}{ Str } & $S a l$ & 0.77 \\
\hline \multirow{2}{*}{ Ssk } & $S c i$ & -0.79 \\
\hline \multirow{2}{*}{ Svk } & $S k u$ & -0.92 \\
\hline \multirow{2}{*}{ Std } & $S f d$ & 0.91 \\
\hline Svi & $S q$ & 0.96 \\
\hline Sp & $S T p$ & 0.77 \\
\hline Sds & $S P c$ & -0.72 \\
\hline Sr1 & $S m v r$ & -0.86 \\
\hline & - & - \\
\hline
\end{tabular}

For some above mentioned representative parameters, but also for parameters with poor correlation coefficient, the calculation procedure was performed according to the methodology given in [32], [33]. This allowed us to obtain the regression equation, which defines the influence of the input parameters of the slide burnishing process on the obtained surface texture parameters. Regression equation coefficients were calculated and then their relevance and repeatability of experimental results were assessed. Adequacy of the obtained regression equation has been rated at the end. This calculation cycle has been performed repeatedly, each time for the different parameters from the above listed surface texture parameters. The result is a few mathematical models in the form of polynomials of the second degree. These models not only allow us to determine the direct influence of input parameters of the slide diamond burnishing process, but also allow us to observe the effects of interactions (synergy) of these parameters. Following mathematical relations have been obtained:
- influence of the slide diamond burnishing parameters on the arithmetical mean height:

$$
\begin{aligned}
S a= & 0.624-0.371 r+0.002 P+2.887 f+0.054 r^{2} \\
& -0.029 P f
\end{aligned}
$$

- influence of the slide diamond burnishing parameters on the root-mean-square height:

$$
S q=1.651-0.739 r-0.0017 P+0.123 r^{2}
$$

- influence of the slide diamond burnishing parameters on the total height:

$$
\begin{aligned}
S t= & 13.546+3.118 r-0.228 P+133.625 f \\
& +0.0009 P^{2}-44.542 r f
\end{aligned}
$$

- influence of the slide diamond burnishing parameters on the texture direction of the surface:

$$
S t d=-66.234+101.377 r-1.219 P-15.007 r^{2}+0.0061 P
$$

- influence of the slide diamond burnishing parameters on the texture aspect ratio:

$$
\text { Str }=0.587-0.002 P
$$

- influence of the slide diamond burnishing parameters on the root mean square gradient of the surface:

$$
\begin{aligned}
S d q= & 0.150-0.103 r+0.0004 P+0.662 f+0.019 r^{2} \\
& -2.503 f^{2}-0.0001 r P-0.065 r f-0.0017 P f
\end{aligned}
$$

- influence of the diamond burnishing parameters on the arithmetic mean summit curvature of the surface:

$$
\begin{aligned}
S S C= & 0.019-0.12 r+0.0001 P+0.071 f+0.002 r^{2} \\
& -0.326 f^{2}-0.00001 r P-0.01075 r f-0.0006 P f
\end{aligned}
$$

- influence of the slide diamond burnishing parameters on the kurtosis of the surface:

$$
\begin{aligned}
S k u= & -129.290+233.738 r-1.444 P-260.347 f \\
& -44.767 r^{2}+0.349 r P
\end{aligned}
$$

- influence of the slide diamond burnishing parameters on the skewness of the surface:

$$
\begin{aligned}
S s k= & 4.196-12.553 r+0.224 P-14.267 f+1.702 r^{2} \\
& -0.034 r P+44.979 r f-1.207 P f
\end{aligned}
$$

Experiments have shown that the slide diamond burnishing process is an effective method of treatment of the parts made of 317Ti stainless steel. It allows us to obtain not 
only a very smooth surface, but also other advantageous features of surface texture without any difficulties. For all obtained dependencies with assumed experimental implementation conditions, all received equations except one, are non-linear. The only linear equation is a model describing the relationship between the input parameters of slide diamond burnishing process and surface texture Str parameter. The calculated regression equations are useful for further analysis (e.g., optimization) and allow for selection of input parameters to obtain a surface texture with desired characteristics during the slide diamond burnishing.

\section{CONCLUSIONS}

1. Slide diamond burnishing process is a relatively easy method to obtain a good surface smoothness of parts made of 317Ti stainless steel.

2. For the comprehensive assessment of the state of the surface texture after the slide roller burnishing process it is enough to measure 10 representative parameters. The other parameters are relatively well correlated with them.

3. The influence of the slide diamond burnishing process input parameters on the surface texture parameters was described by the obtained regression equations. It allowed the disclosure of the synergies of individual parameters of the slide diamond burnishing process.

\section{REFERENCES}

[1] Oczos, K.E., Liubimov, V. (2003). Struktura geometryczna powierzchni [Surface Geometric Structure]. Rzeszow, Poland: Rzeszow Technical University Publishing House. (in Polish)

[2] Berglund, J., Brown C.A., Rose, B.G., Bay, N. (2010). Milled die steel surface roughness correlation with steel sheet friction. CIRP Annals - Manufacturing Technology, 59, 577-580.

[3] Sedlacek, M., Podgornik, B., Vizintin, J. (2012). Correlation between standard roughness parameters skewness and kurtosis and tribological behaviour of contact surfaces. Tribology International, 48, 102-112.

[4] Stout, K., Davis, E.I., Sullivan, P.I. (1990). Atlas of Machined Surfaces. Chapman and Hall.

[5] Stout, K.J., Dong, W.P., Mainsah, E. (1993). A proposal for standardization of assessment of threedimensional micro-topography - Part 1. Surface digitisation and parametric characterisation. University of Birmingham.

[6] Thomas, T.R. (1999). Rough Surfaces. Imperial College Press.

[7] Niemczewska-Wójcik, M. (2017). Multi-sensor measurements of titanium alloy surface texture formed at subsequent operations of precision machining process. Measurement, 96, 8-17.

[8] Low, K.O. (2011). Surface characteristics modification of polyoxymethylene and polyurethane using burnishing. Tribology Transactions, 54 (1), 96-103.

[9] Yusof, N.F.M., Ripin, Z.M. (2014). Analysis of surface parameters and vibration of roller bearing. Tribology Transactions, 57 (4), 715-729.
[10] Coutinho, R., Marinescu, I.D. (2005). Methodology to compare 3-D and 2-D parameters for the optimization of hard turned surfaces. Machining Science and Technology, 9 (3), 383-409.

[11] Wang, G., Zhou, X., Meng, G., Yang, X. (2017). Modeling surface roughness for polishing process based on abrasive cutting and probability theory. Machining Science and Technology, 22 (1), 86-98.

[12] Grzesik, W. (2016). Prediction of the functional performance of machined components based on surface topography: State of the art. Journal of Materials Engineering and Performance, 25 (10), 4460-4468.

[13] Wagner, J.J., Jenson, A.D., Sundararajan, S. (2017). The effect of contact pressure and surface texture on running-in behavior of case carburized steel under boundary lubrication. Wear, 376-377, 851-857.

[14] Krzyzak, Z., Pawlus, P. (2006). 'Zero-wear' of piston skirt surface topography. Wear, 260, 554-561.

[15] Profito, F.J., Vlădescu, S-C., Reddyhoff, T., Dini, D. (2017). Transient experimental and modelling studies of laser-textured microgrooved surfaces with a focus on piston-ring cylinder liner contacts. Tribology International, 113, 125-136.

[16] Khelifi, C., Do, M.T., Kane, M., Adenot Meyer, M. (2017). Wear and wet friction of steel tracks for rubber-tired metros. Wear, 376-377, 1912-1918.

[17] Niemczewska-Wójcik, M. (2016). Multi-sensor measurements of titanium alloy surface texture formed at subsequent operations of precision machining process. Measurement, 96, 8-17.

[18] Kang, Y.S., Hager, C.H., Evans, R.D. (2015). Effects of skewed surface textures on lubricant film thickness and traction. Tribology Transactions, 58 (3), 397-406.

[19] Gherca, A., Fatu, A., Hajjam, M., Maspeyrot, P. (2013). Influence of surface geometry on the hydrodynamic performances of parallel bearings in transient flow conditions. Tribology Transactions, 56 (6), 953-967.

[20] Sedlaček, M., Gregorčič, P., Podgornik, B. (2017). Use of the roughness parameters Ssk and Sku to control friction-a method for designing surface texturing. Tribology Transactions, 60 (2), 260-266.

[21] Wang, L., Ouyang, W., Gao, W., Xu, B. (2017). Instrumental evaluation of fabric abrasive wear using 3D surface images. Journal of the Textile Institute, 108 (5), 846-851.

[22] Lu, W., Zhang, G., Liu, X., Zhou, L., Chen, L., Jiang, X. (2014). Prediction of surface topography at the end of sliding running-in wear based on areal surface parameters. Tribology Transactions, 57 (3), 553-560.

[23] Masuko, M., Aoki, S., Suzuki, A. (2005). Influence of lubricant additive and surface texture on the sliding friction characteristics of steel under varying speeds ranging from ultralow to moderate. Tribology Transactions, 48 (3), 289-298.

[24] Eiss, N.S., Bayraktaroglu, M.M. (1980). The effect of surface roughness on the wear of low-density polyethylene. ASLE Transactions, 23 (3), 269-278. 
[25] Shi, X., Wang, L., Qin, F. (2016). Relative fatigue life prediction of high-speed and heavy-load ball bearing based on surface texture. Tribology International, 10, 364-374.

[26] Trauth, D., Klocke, F., Welling, D., Terhorst, M., Mattfeld, P., Klink, A. (2016). Investigation of the surface integrity and fatigue strength of Inconel718 after wire EDM and machine hammer. International Journal of Material Forming, 9 (5), 635-651.

[27] Qi, Q., Li, T., Scott, P.J., Jiang, X. (2015). A correlational study of areal surface texture parameters on some typical machined surfaces. Procedia CIRP, 27, 149-154.

[28] Rosen, B.G., Anderberg, C., Ohlsson, R. (2008). Parameter correlation study of cylinder liner roughness for production and quality control. Proceedings of the Institution of Mechanical Engineers, Part B: Journal of Engineering Manufacture, 222, 1475-1487.

[29] Das, J., Linke, B. (2017). Evaluation and systematic selection of significant multi-scale surface roughness parameters (SRPs) as process monitoring index.
Journal of Materials Processing Technology, 244, 157-165.

[30] Franco, L.A., Sinatora, A. (2015). 3D surface parameters (ISO 25178-2): Actual meaning of Spk and its relationship to Vmp. Precision Engineering, 40, 106-111.

[31] Korzynski, M. (2013). Slide diamond burnishing. In Nonconventional Finishing Technologies. Warsaw, Poland: Polish Scientific Publishers PWN.

[32] Korzynski, M. (2017). Metodyka eksperymentu [Methodology of Experiment]. Warsaw, Poland: Science Publishing Home. (in Polish)

[33] Korzynski, M., Lubas, J., Swirad, S., Dudek, K. (2011). Surface layer characteristics due to slide diamond burnishing with a cylindrical-ended tool. Journal of Materials Processing Technology, 211 (1), 84-94.

Received December 12, 2017. Accepted May 14, 2018. 